\title{
Investigation of the role of type VII collagen in the HIF1- alpha induced pro-angiogenic pathway
}

\author{
F Shams, ${ }^{1}$ M. Krupiczojc, ${ }^{1}$ S. Alexander, ${ }^{1}$ M Caley, ${ }^{1}$ and EA O'Toole. ${ }^{1}$
}

1 Centre for Cell Biology and Cutaneous Research, Barts and the London School of Medicine and Dentistry, London, United Kingdom

\section{Background}

Tvpe VII collagen (Col7) is the main component of anchoring fibrils in the basement membrane of skin. Loss of function mutations in the COL7A1 gene lead to recessive dystrophic epidermolysis bullosa (RDEB) with severe congenital blistering, scarring and later development of aggressive squamous cell carcinomas (SCCS). A recent study from our group has shown an increase in angiogenesis with loss of Col7 in RDEB. One of the main regulators of tumour angiogenesis is hypoxia. The most important regulator of the cellular response to hypoxia is the transcription factor HIF-1 and its subunit HIF-I alpha (HIF-1a). In this study, we investigated the effect of hypoxia on SCC cell lines with loss of Col7 on HIF-1 $\alpha$ and angiogenic factors VEGF, Amphiregulin, Urokinase Plasminogen Activator and Ephrin-A1

\section{Results}

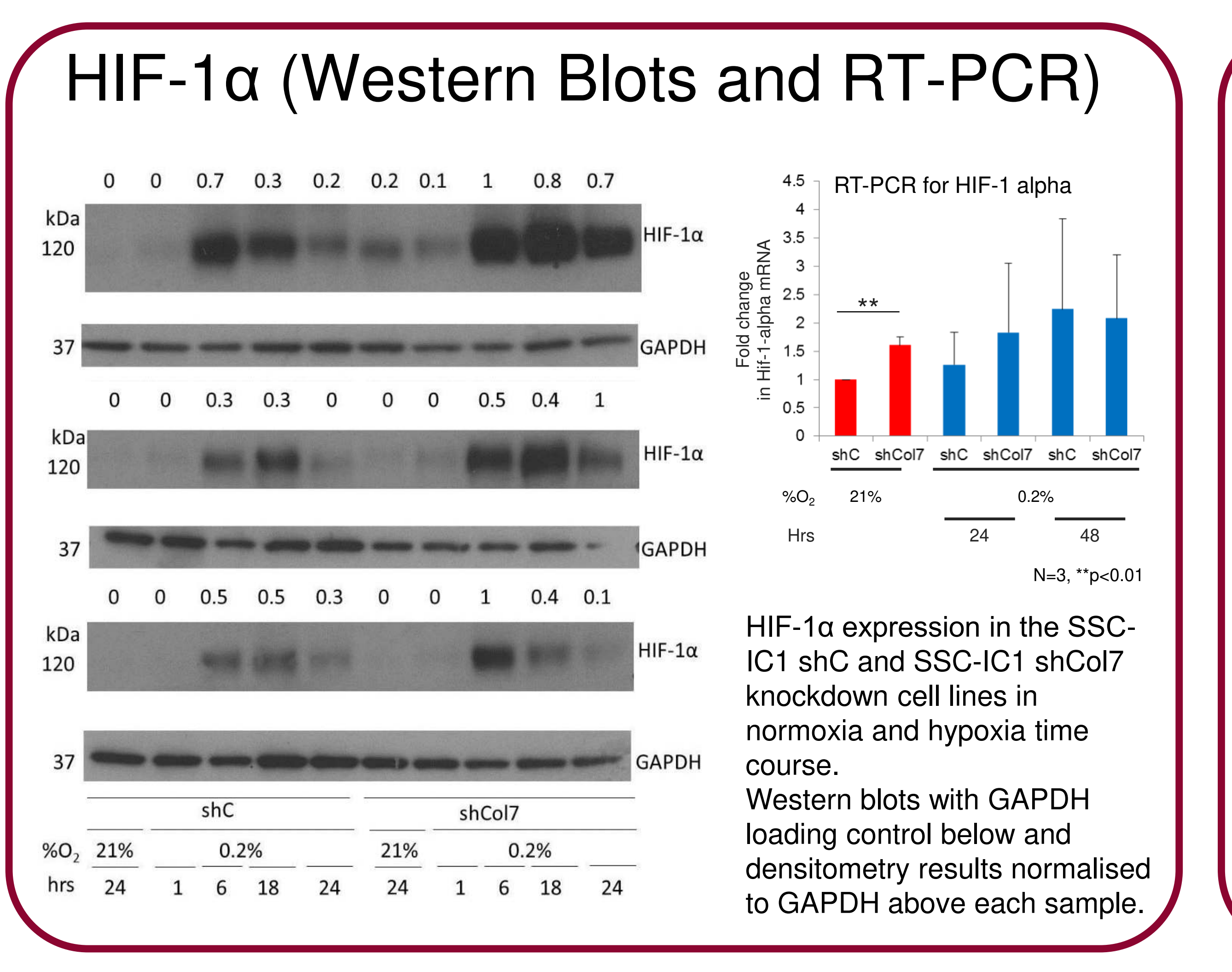

Angiogenic Factors Western Blots

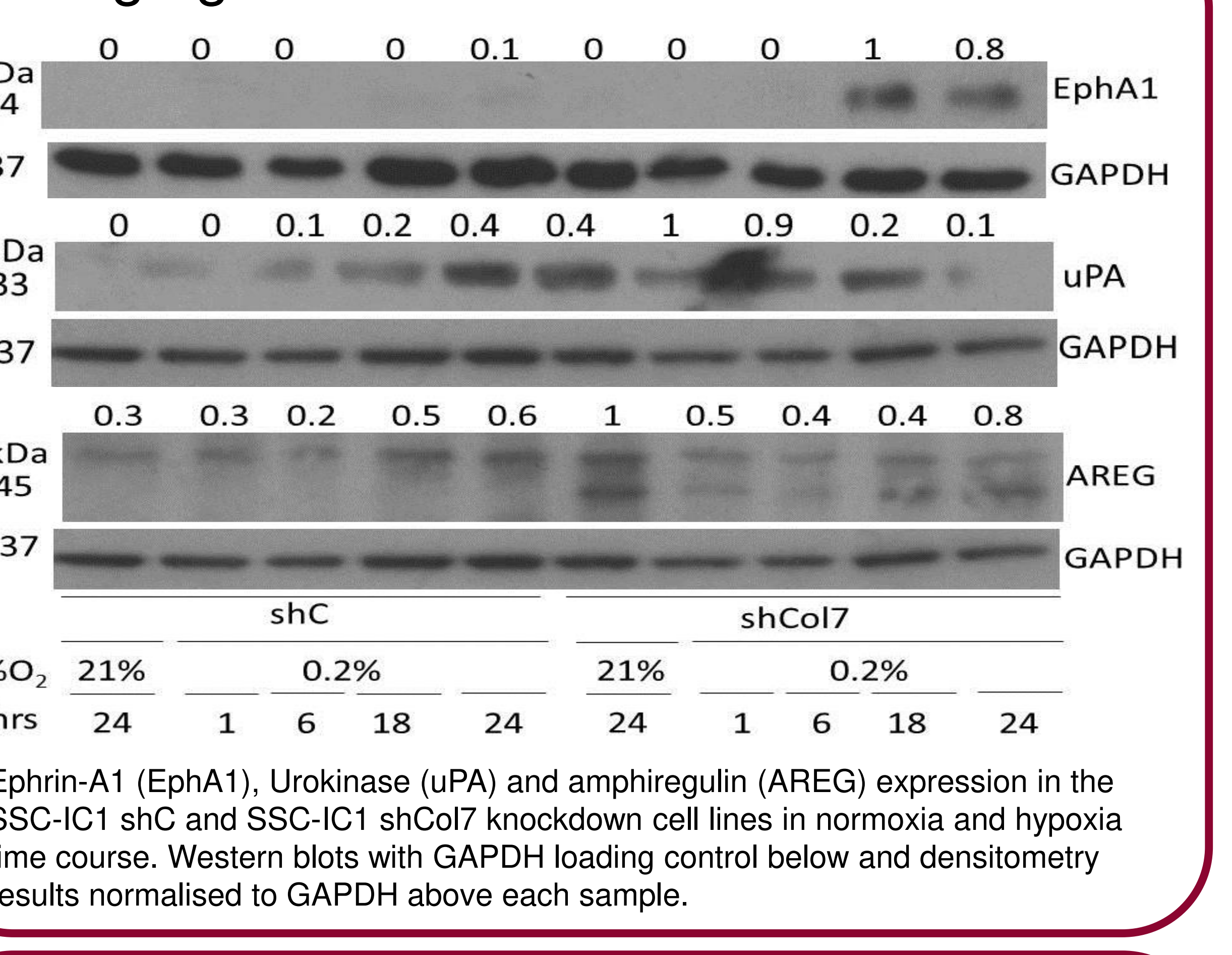

uPA Immunofluorescence Staining
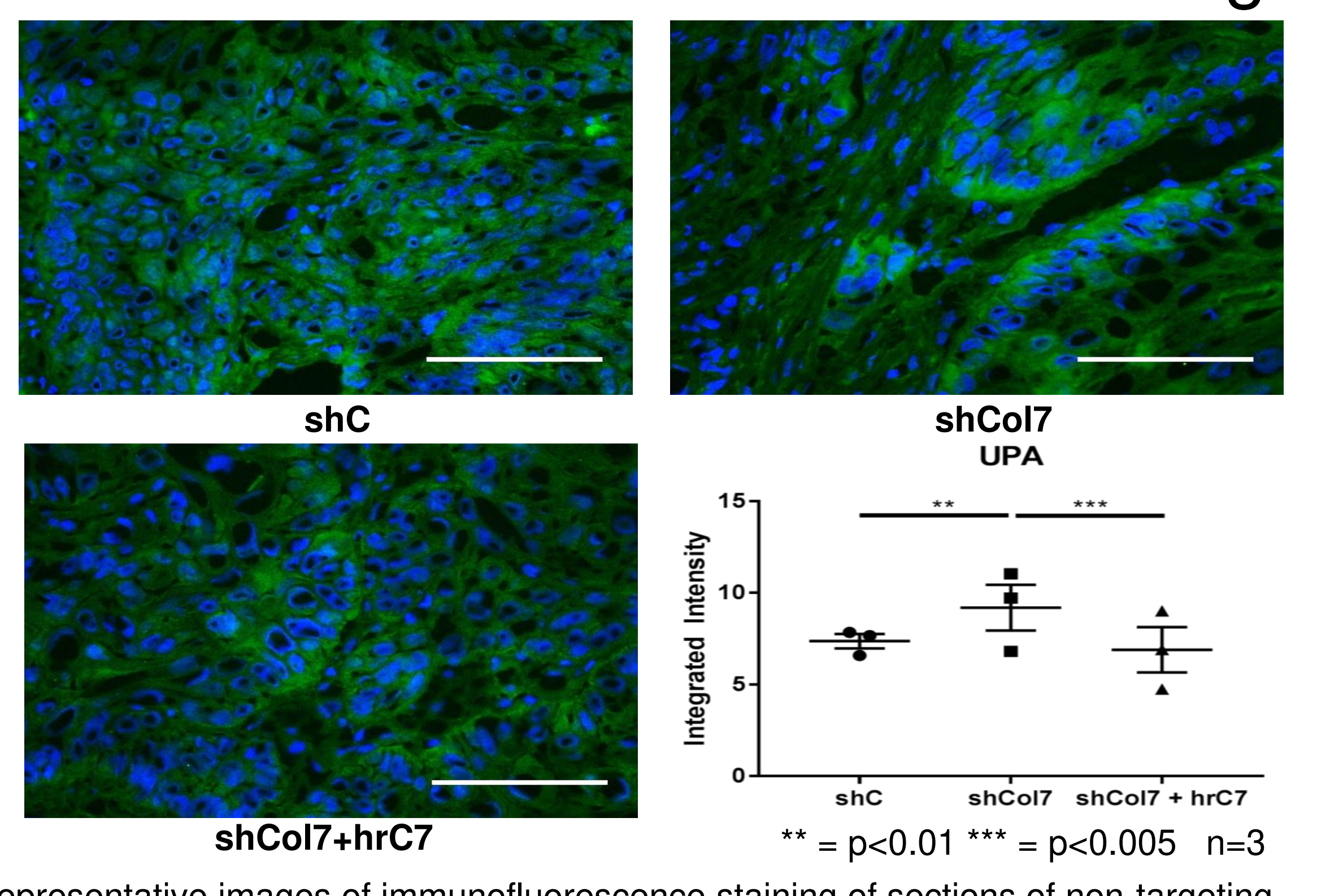

shCol7 +hrC7

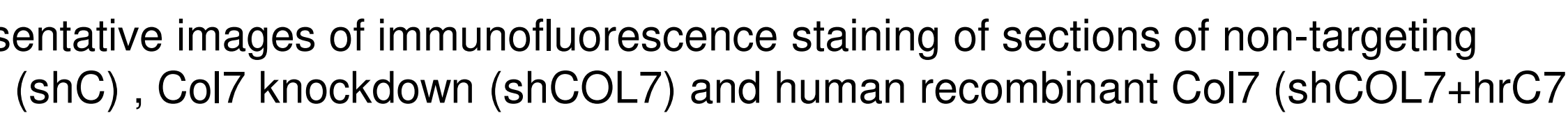

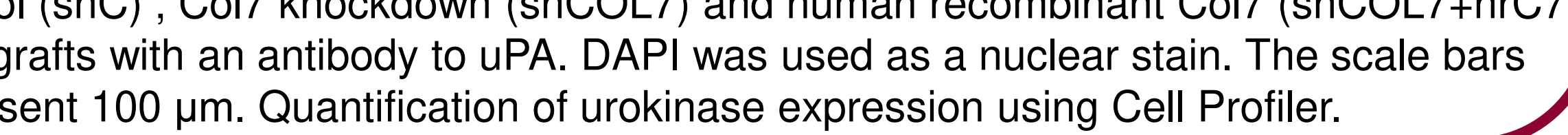

\section{- Loss of Col7 enhances hypoxic signals.}

- Loss of Col7 enhances the expression of pro-angiogenic factors, VEGF, VEGFR1, Amphiregulin, Urokinase and Ephrin-A1

- Reintroduction of Col7 reduces the enhancement of expression of hypoxic marker and pro-angiogenic factors.

- This work improves our understanding of angiogenesis in RDEB.

- These findings suggest that anti-angiogenic therapy may be beneficial to RDEB patients.

- This could lead to new therapeutic strategies for RDEB wounds and SCC targeting angiogenic pathways and the adaptive hypoxic response activated by loss of Col7.
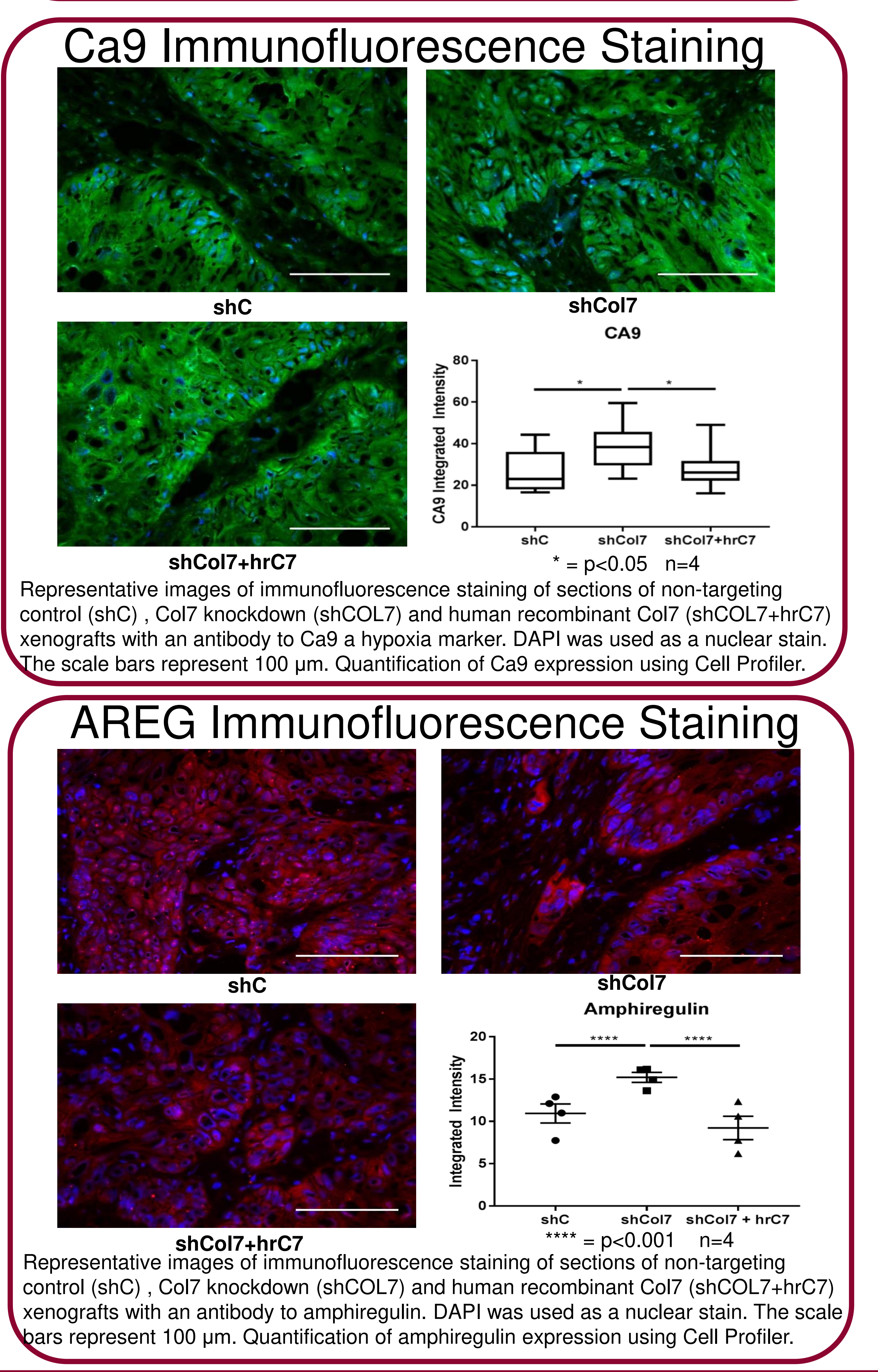

Conclusions
Angiogenic Factors qPCR

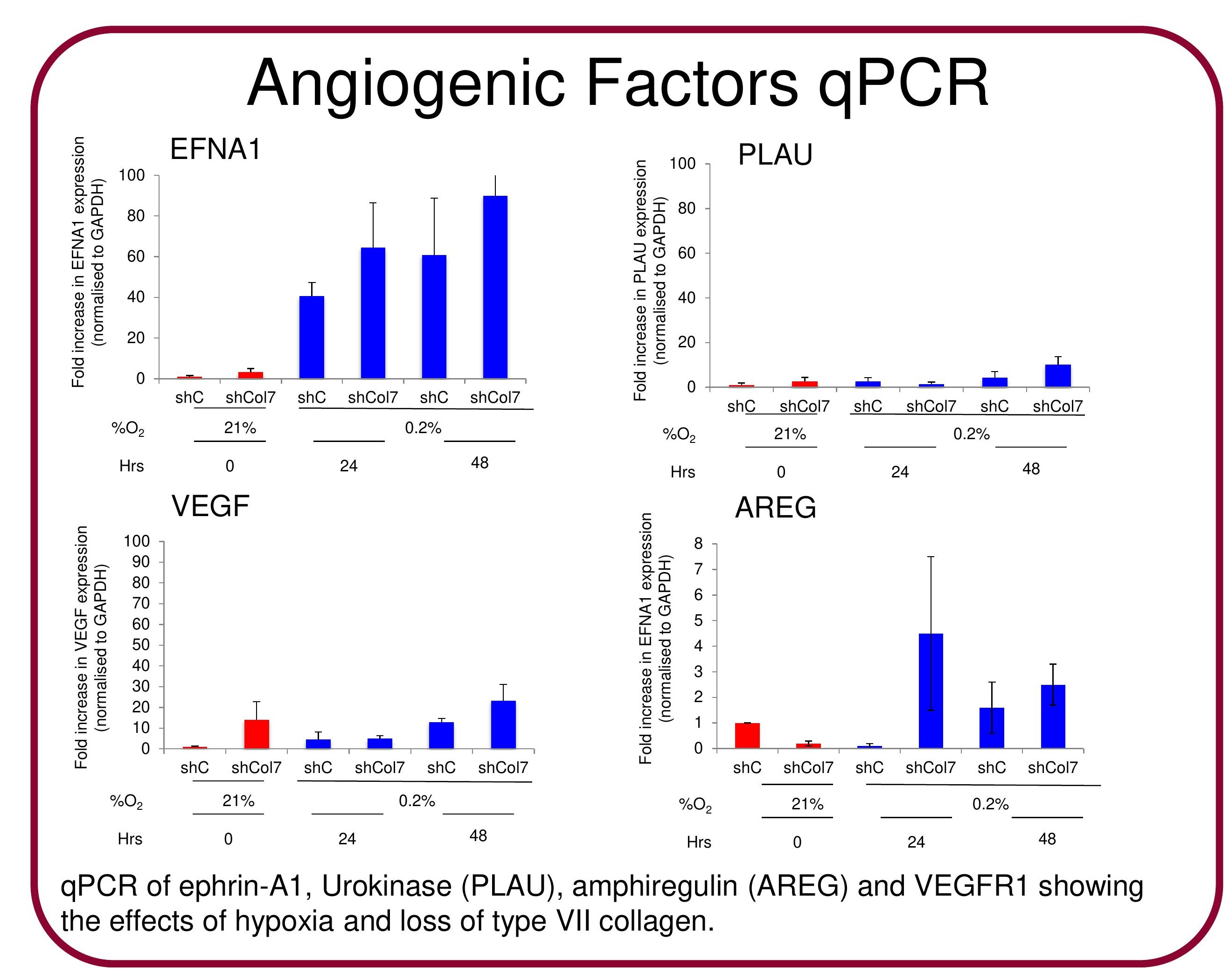

Ephrin-A1 Immunofluorescence Staining
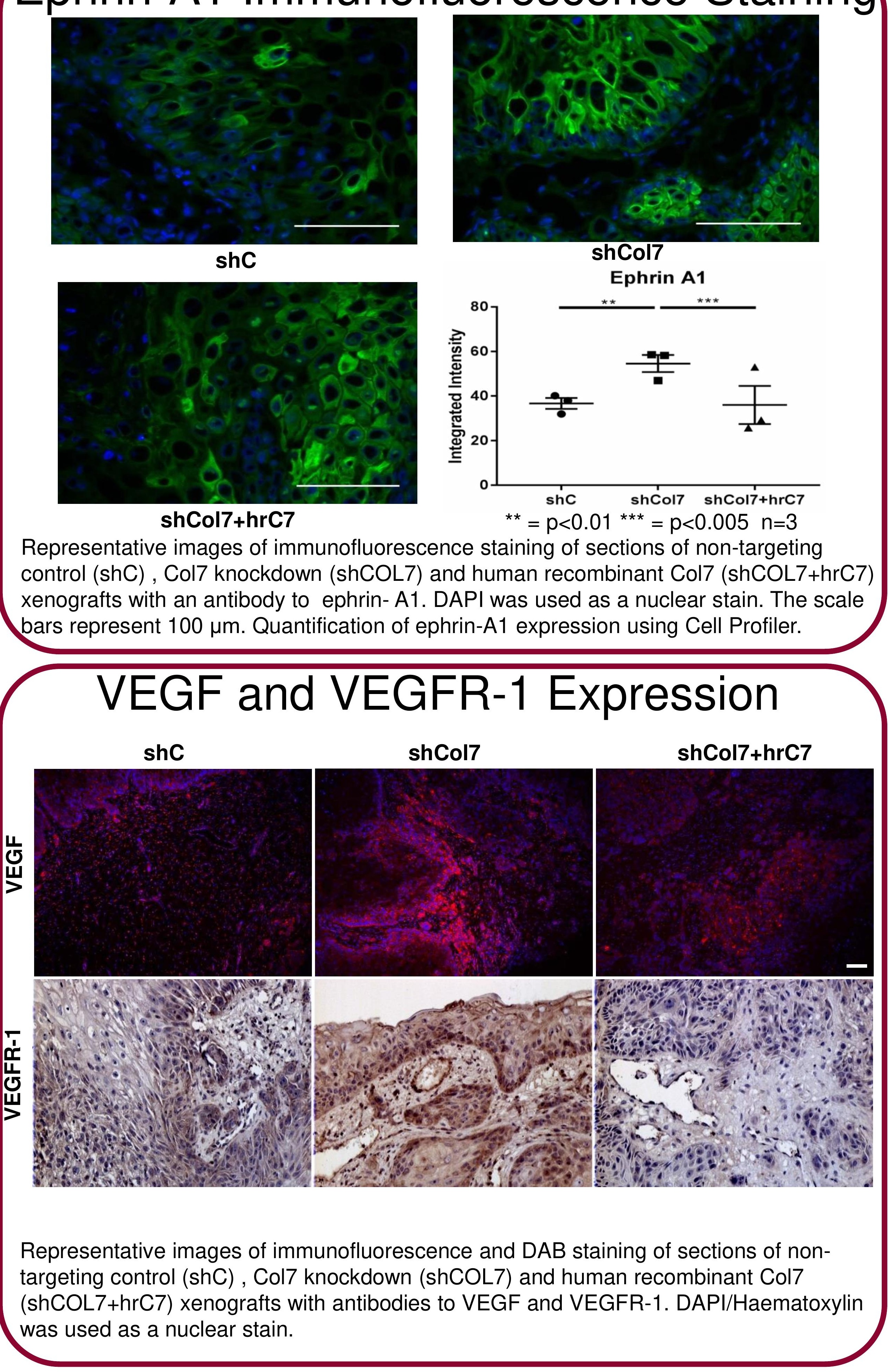

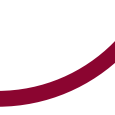

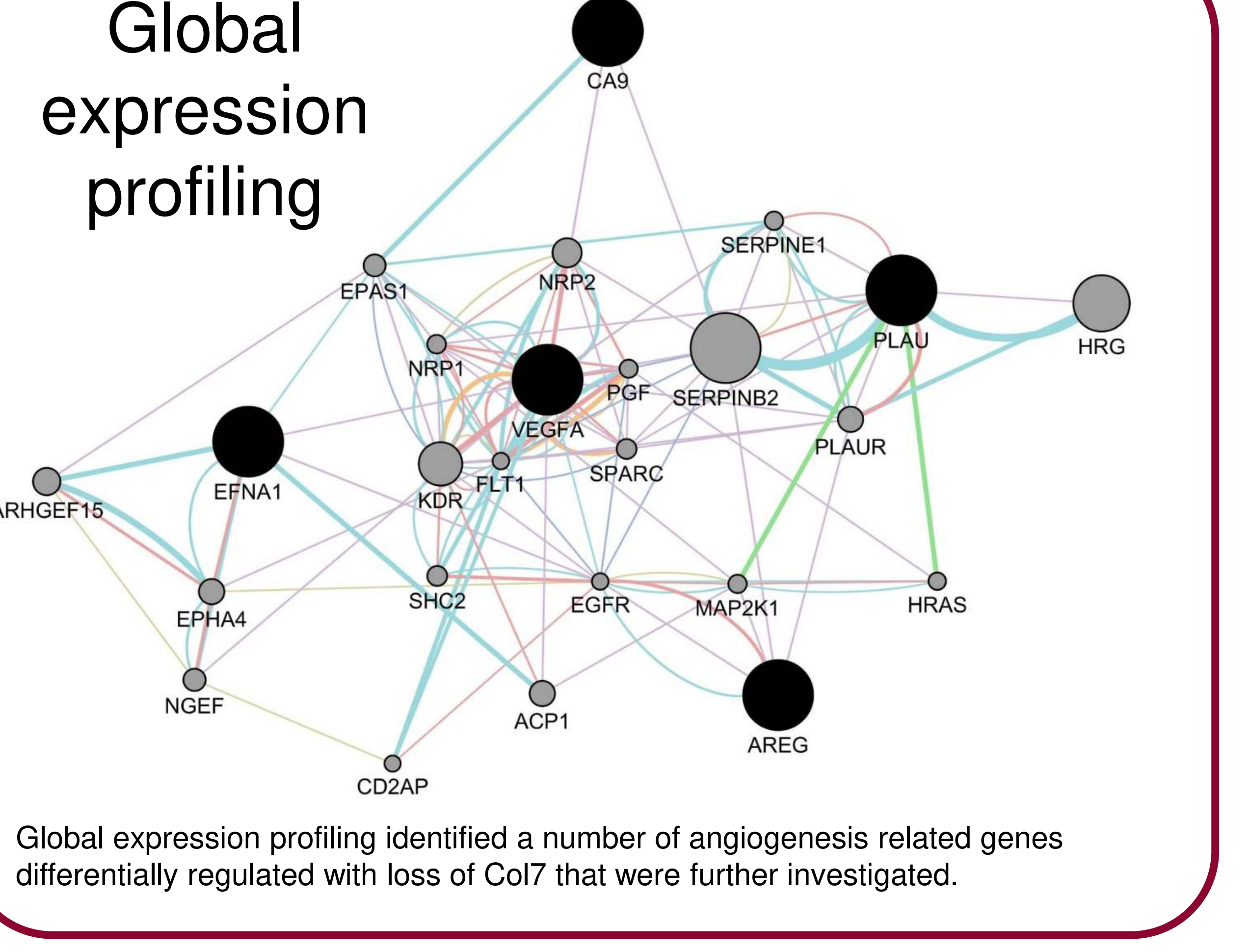
0 EO C 0 skin doesn't work 